\title{
Metal-Based Nanoparticles Enhance Drought Tolerance in Soybean
}

\author{
Tran My Linh $\left(\mathbb{D},{ }^{1}\right.$ Nguyen Chi Mai $\mathbb{D}^{1},{ }^{1}$ Pham Thi Hoe $\mathbb{D}^{1},{ }^{1}$ Le Quynh Lien $\mathbb{D}^{1},{ }^{1}$ \\ Ninh Khac Ban, ${ }^{1}$ Le Thi Thu Hien $\left(1,{ }^{2}\right.$ Nguyen Hoai Chau $\mathbb{D}^{3},{ }^{3}$ and Nguyen Tuong $\operatorname{Van}^{4}$ \\ ${ }^{1}$ Institute of Marine Biochemistry, Vietnam Academy of Science and Technology (VAST), 18 Hoang Quoc Viet, Cau Giay, \\ Hanoi, Vietnam \\ ${ }^{2}$ Institute of Genome Research, VAST, 18 Hoang Quoc Viet, Cau Giay, Hanoi, Vietnam \\ ${ }^{3}$ Institute of Environmental Technology, VAST, 18 Hoang Quoc Viet, Cau Giay, Hanoi, Vietnam \\ ${ }^{4}$ Institute of Biotechnology, VAST, 18 Hoang Quoc Viet, Cau Giay, Hanoi, Vietnam \\ Correspondence should be addressed to Tran My Linh; tmlinh@imbc.vast.vn
}

Received 3 January 2020; Revised 5 May 2020; Accepted 12 May 2020; Published 20 June 2020

Academic Editor: Enrico Bergamaschi

Copyright (c) 2020 Tran My Linh et al. This is an open access article distributed under the Creative Commons Attribution License, which permits unrestricted use, distribution, and reproduction in any medium, provided the original work is properly cited.

Drought is a major abiotic stress that negatively impacts plant growth and crop production. Among various techniques used to alleviate drought stress in plants, nanoparticle application is considered to be effective and promising. In this study, the responses of plants treated with iron, copper, cobalt, and zinc oxide nanoparticles (NPs) were analyzed in soybean under drought-induced conditions. The obtained results indicated that these metal-based NPs supported the drought tolerance of NP-treated plants. The desired physiological traits, viz., relative water content, drought tolerance index, and biomass reduction rate, were significantly improved, especially in iron NP-treated plants. At the molecular level, quantitative PCR analysis of several drought-responsive genes revealed a gene-, tissue-, and NP-dependent upregulation of gene expression. Iron NP treatment promoted the expression of all tested genes in roots; additionally, the expression of three droughtresponsive genes increased in leaves of all NP-treated plants, while the expression of GmERD1 (Early Responsive to Dehydration 1) was induced in both roots and shoots under the four NP treatments tested. Our findings suggest that NP application can improve drought tolerance of soybean plants by triggering drought-associated gene expression.

\section{Introduction}

Among the top 10 most widely grown crop species, soybean (Glycine max (L.) Merrill) is a globally important crop used for both food and animal feed [1]. Unfortunately, it is also among the most drought-susceptible crop plants [2], with up to $40 \%$ of annual yield losses due to water shortage. The problem is even more serious in tropical countries, such as Vietnam, where the prevalence and duration of drought have increased over the years. To overcome this challenge, alternative breeding and biotechnological approaches, such as micronutrient modification, have been extensively studied [3-6]. The supplementation of mineral microelements, such as iron, copper, cobalt, magnesium, manganese, nickel, and zinc, is known to increase the crop yield, especially under adverse environmental conditions $[7,8]$.

The rapid development of nanotechnology has allowed metal-based nanoparticles (NPs) of 1-100 nm in size and in extremely low quantity to be tested as substitute plant mineral nutrients and stimulants. The application of NPs enhances plant responses to drought stress compared to conventional bulk fertilization forms [9]. For example, the application of $\mathrm{ZnO}$ NPs increased soybean seed germination percentage under water stress [10]. Similarly, sorghum productivity and nitrogen acquisition improved remarkably with $\mathrm{ZnO} \mathrm{NP}$ application to the soil [11]. Furthermore, the addition of $\mathrm{ZnO}$ NPs to the culture medium promoted somatic embryo formation and plant regeneration and increased 
stress tolerance in in vitro-grown banana plants [12]. In turn, $\mathrm{Cu}$ and $\mathrm{Zn}$ NPs effectively attenuated drought effects on wheat plants by increasing antioxidant enzyme activities and relative water content, reducing thiobarbituric acid reactive substance (TBARS) accumulation, and stabilizing photosynthetic pigment content in leaves [13]. The use of zerovalent Fe NPs was found to support normal drought sensitivity maintenance, plasma membrane $\mathrm{Hp}$-ATPase activation, and stomatal opening, as well as chlorophyll and plant biomass content, while $\mathrm{CO}_{2}$ assimilation of Arabidopsis thaliana increased during water stress [14].

Although the possible roles of NPs in boosting plant defense responses to abiotic stress, including water stress, have been extensively studied, many aspects remain to be elucidated. When analyzing related researches and their own work, Khan et al. [15] hypothesized that NPs may mediate plant adaption and tolerance to stress through the activation of defense systems and stress-related gene expression. Plants respond to environment stress factors by activating various transcellular membrane sensors, particularly $\mathrm{Ca}^{2+}$ channels and $\mathrm{Ca}^{2+}$-binding proteins [16]. Subsequent downstream events lead to changes in gene expression and ultimately to plant adaptation to stress. Among stress-responsive genes, RD20A (Response to Desiccation 20A) and ERD1 (Early Responsive to Dehydration 1) are among the most responsive stress marker genes in plants, including soybean $[17,18]$. Regarding transcription factor (TF) genes, families such as bZIP (basic leucine zipper), DREB (dehydration-responsive element-binding factor), NAC (NAM (no apical meristem), ATAF (Arabidopsis transcription activation factor), and CUC (cup-shaped cotyledon)), WRKY (composed of a conserved WRKYGQK motif), MYB (myeloblastosis), ERF (ethylene response factor), and ABF/AREB (abscisic acidresponsive element-binding factor) are associated with plant adaptation to environmental stress $[19,20]$. The overexpression of GmFDL19, of the bZIP family, significantly improved transgenic soybean tolerance to water shortage [21]. Similarly, GmDREB2 overexpression led to enhanced drought and high-salt stress tolerance of transgenic Arabidopsis plants [22]. Recently, GmWRKY27, GmMYB118, and $G m M Y B 174$ were found to be strongly upregulated in soybean during abiotic stress, including drought $[23,24]$. Furthermore, GmNAC11 regulated DREB1A and other stressrelated genes (COR15A,ERF5, ERD11, etc.) in transgenic soybean [25].

The contribution of metal-based NPs to the improvement of plant adaptation to abiotic stress has been extensively demonstrated[26-32]; however, their role in inducing stress-related gene expression has not been evaluated yet. Therefore, in the present study, our objective was to evaluate physiological and molecular responses of soybean plants treated with $\mathrm{Fe}, \mathrm{Cu}, \mathrm{Co}$, and $\mathrm{ZnO}$ NPs under water stress. Physiological indicators studied included relative water content (RWC), drought tolerance index (DTI), and biomass reduction rate. As for the expression of drought tolerance marker genes, our analysis included $G m R D 20 A$, GmDREB2, GmERD1, GmFDL19, GmNAC11, GmWRKY27, $G m M Y B 118$, and GmMYB174. A model explaining the likely mode of action of NPs in triggering plant tolerance to water stress was proposed based on the results reported herein and the literature available.

\section{Materials and Methods}

2.1. Sample Preparation and Drought Treatments. Seeds of the local soybean (Glycine max (L.) Merrill) cultivar DT26 harvested in the winter of 2017 were provided by the Legumes Research and Development Center of the Food Crops Research Institute at the Vietnam Academy of Agriculture Sciences. Healthy, uniformly sized seeds were selected for NP seed treatment. NPs, including $\mathrm{Fe}, \mathrm{Cu}, \mathrm{Co}$, and $\mathrm{ZnO}$ with purity $>95 \%$ and size of $20-60 \mathrm{~nm}$, provided by the Institute of Environmental Technology, VAST [33], were suspended in distilled deionized water separately to generate $50 \mathrm{mg} / \mathrm{L}$ of $\mathrm{Fe}, \mathrm{ZnO}$, and $\mathrm{Cu}$ NPs and $0.05 \mathrm{mg} / \mathrm{L} \mathrm{Co} \mathrm{NP}$; subsequently, each suspension was sonicated with Elmasonic $\mathrm{S}$ 100/H (Elma Schmidbauer GmbH, Singen, Germany) at $200 \mathrm{~W}$ and $37 \mathrm{kHz}$ for $30 \mathrm{~min}$ and immediately used for seed treatment. One hundred and fifty seeds (approximately $30 \mathrm{~g}$ ) were soaked in $10 \mathrm{~mL}$ NP suspensions for $30 \mathrm{~min}$. The control batches of seeds were soaked in distilled deionized water. The concentrations of each NP type were selected based on our previous experimental results [34].

Drought treatment was carried out as described by Thu et al. [35]. Briefly, in all experiments, plants were grown in a premixed, standard clean black soil (Namix, Ho Chi Minh City, Vietnam) rich in nutrients and germ-free. Control (untreated) and NP-treated seeds were sown in PVC sheets rolled into tubular shape $(80 \mathrm{~cm}$ in height, $10 \mathrm{~cm}$ in diameter, 1 seed/tube) for maximum axial root growth. Plants were grown in a net house under natural light, $28-30^{\circ} \mathrm{C}$ ambient temperature, and $60-70 \%$ relative humidity; they were watered every day until the V2-V3 (vegetative second and third nodes) stage (12 days) and then divided into wellwatered control and induced drought stress groups for which watering was completely stopped for the following 15 days. Soil moisture content (SMC) was monitored at 5-day intervals $(n=2)$ using the HydroSense II system (Campbell Scientific, Inc., Logan, UT, USA) to maintain approximately $65-70 \%$ SMC for the control group while for the induced drought stress treatment groups, SWC was allowed to gradually decrease to $30-40 \%$ by the end of the experimental period.

After drought treatment, plants $(n=20$ for each treatment) were gently removed and the lengths of shoots and roots of each individual plant were recorded. Roots and shoots were then dried separately at $65^{\circ} \mathrm{C}$ for $48 \mathrm{~h}$ to determine dry weight (DW). The specific root length (SRL) index was calculated based on root length and dry mass.

\subsection{Physiological Parameter Assays}

2.2.1. Relative Water Content. Determination of relative water content (RWC) was carried out 27 days after planting as described by Hossain et al. [36]. The aerial parts of the plants $(n=20)$ from NP-treated and nontreated groups were collected and weighed immediately for calculating fresh weight (FW). Turgid weight (TW) of samples was estimated by soaking samples in deionized water for $24 \mathrm{~h}$ at $26 \pm 1^{\circ} \mathrm{C}$, 
TAble 1: Primer sequences used in qPCR analysis.

\begin{tabular}{lccccc}
\hline $\mathrm{Nr}$ & Gene & Forward sequence $\left(5^{\prime}-3^{\prime}\right)$ & Reverse sequence $\left(5^{\prime}-3^{\prime}\right)$ & Amplicon length (bp) & Ref. \\
\hline 1 & GmRD20A & GTGGCACATGACTGAAGGAA & ATCTTTCCAGCAGCACCTCT & 195 & {$[18]$} \\
2 & GmDREB2 & GAAAGCAGCAGCACCAACAA & GGCGTAAGAACCCAACCAGA & 125 & This study \\
3 & GmERD1 & CGTCCAGAATTGCTCAACAG & TGGGGTTATAGCCTTGTTGG & 184 & {$[18]$} \\
4 & GmFDL19 & GGTTTGGAGAGATATGCAAC & GGCATGTTGTGATGTGTTGT & 181 & {$[21]$} \\
5 & GmNAC11 & TGCAAGGAGGAGCACAAGAG & CACAGAACCCAGTCGTCCAA & 175 & This study \\
6 & GmWRKY27 & CATTGGATTTGGAGGTGAAGA & TCTCTGTGAGCATTTCGGTTA & 112 & This study \\
7 & GmMYB118 & TTGCTTGAGATTATGGTCCTTG & CATCACCCTTTCCTTCAACC & 113 & This study \\
8 & GmMYB174 & TGGCATAAAATAGGGAGCTGA & AAAAGGCAAGTTCACGGATT & 90 & This study \\
9 & GmFbox & AGATAGGGAAATTGTGCAGGT & CTAATGGCAATTGCAGCTCTC & 93 & {$[38]$} \\
\hline
\end{tabular}

placing them on absorbent paper to remove the surface water, and weighing them. Finally, samples were dried at $65^{\circ} \mathrm{C}$ for $48 \mathrm{~h}$ for dry weight (DW) measurements. RWC was calculated as per the following formula:

$$
\mathrm{RWC}(\%)=\frac{\mathrm{FW}-\mathrm{DW}}{\mathrm{TW}-\mathrm{DW}} \times 100 .
$$

2.2.2. Drought Tolerance Index. Examination of the drought tolerance index (DTI) was performed as described by Thu et al. [35]. Seeds of each NP treatment and control were germinated in plastic pots $(25 \mathrm{~cm}$ in height and $30 \mathrm{~cm}$ in diameter, five seeds/pot; $n=30$ ) and watered regularly for 12 days. Drought treatment was carried out as described above. During the 15 days of drought treatment duration, the number of nonwithered plants was recorded every two days. When SMC decreased to $30 \%$ or lower and the number of nonwithered plants reached more than $50 \%$ of all plants in the group, plants were watered again for the following 15 days. The number of plants showing recovery was determined at 2-day intervals. DTI of each NP treatment was calculated as per the following formula:

$$
\begin{aligned}
\mathrm{DTI}= & \frac{1}{2} \sin \alpha\left(D_{1} R_{1}+R_{1} D_{3}+D_{3} R_{3}+R_{3} D_{5}+D_{5} R_{5}\right. \\
& \left.+R_{5} D_{7}+\cdots+D_{15} R_{15}+R_{15} D_{1}\right),
\end{aligned}
$$

where $D_{n}$ is the percentage of nonwilted plants after $n$ day(s) of drought treatment, $R_{n}$ is the percentage of plants showing recovery after $n$ day(s) of rewatering, $\alpha=360 / 2 n$, and the number of equal inner angles $(2 n)$ is 16 .

2.2.3. Biomass Reduction Rate. Plant biomass reduction under drought was calculated according to Nguyen et al. [37]. Each plant was grown in a plastic pot containing $600 \mathrm{~g}$ soil and $250 \mathrm{~mL}$ water. Experiments involving drought treatment were performed as described above. After 15 days, plants were allowed to recover by rewatering for the following seven days. The entire shoot of each plant was collected and dried at $65^{\circ} \mathrm{C}$ for $48 \mathrm{~h}$ to determine plant dry weight ( $n=10$ per treatment). The biomass reduction rate (\%) was calculated as per the following formula:

$$
\operatorname{Biomass} \text { reduction }(\%)=\frac{\mathrm{WW}-\mathrm{DR}}{\mathrm{WW}} \times 100 \text {, }
$$

where WW is the dry weight of plants under well-watered conditions and DR is the dry weight of plants showing recovery from drought treatment.

2.3. Gene Expression Analyses. To analyze the effects of NP treatment on the expression of target genes under drought conditions, roots and leaves of plants were collected for RNA isolation. Total RNA was isolated and purified using the GeneJET Plant RNA Purification Mini Kit (Thermo Fisher Scientific, Waltham, MA, USA), and DNA was removed by DNaseI using the RapidOut DNA removal kit (Thermo Fisher Scientific, Waltham, MA, USA). RNA concentrations were determined using the NanoDrop ${ }^{\mathrm{TM}}$ 2000/2000c Spectrophotometers (Thermo Fisher Scientific, Waltham, MA, USA). cDNA synthesis was performed using $1 \mu \mathrm{g}$ total RNA and the Maxima First Strand cDNA Synthesis Kit (Thermo Fisher Scientific, Waltham, MA, USA).

Primer pairs for drought-responsive genes GmDREB2, GmNAC11, GmWRKY27, GmMYB118, and GmMYB174 were designed using the Primer3Plus software (http://frodo .wi.mit.edu/primer3/), while primer sequences for the other genes (GmRD20A, GmERD1, and GmFDL19) were designed as reported previously $[18,21]$. Primer specificity was confirmed by BLAST (http://blast.ncbi.nlm.nih.gov/Blast.cgi), followed by analyzing the melting curves and amplicon fragments. The sequences of primers used are listed in Table 1. The GmFbox gene was selected as a reference gene [38].

Reverse transcription- (RT-) qPCR was performed in 96well plates on the Eppendorf Realplex 4 Mastercycler (Eppendorf AG, Hamburg, Germany) using the SYBR Green PCR Master Mix (Thermo Fisher Scientific, Waltham, MA, USA). RT-qPCR reaction was carried out as described by Thao et al. [39]. Relative expression levels of each gene were calculated using the $2^{-\Delta \Delta \mathrm{Ct}}$ method [40] with expression normalized against the internal reference gene, GmFbox. Three independent biological samples were used from each experimental group. 


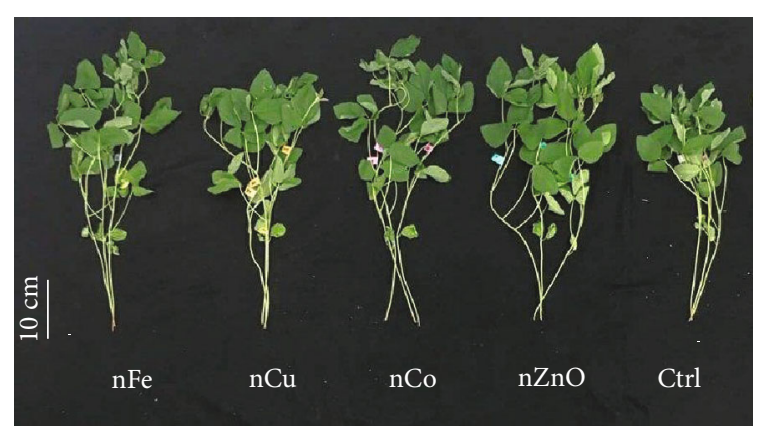

(a)

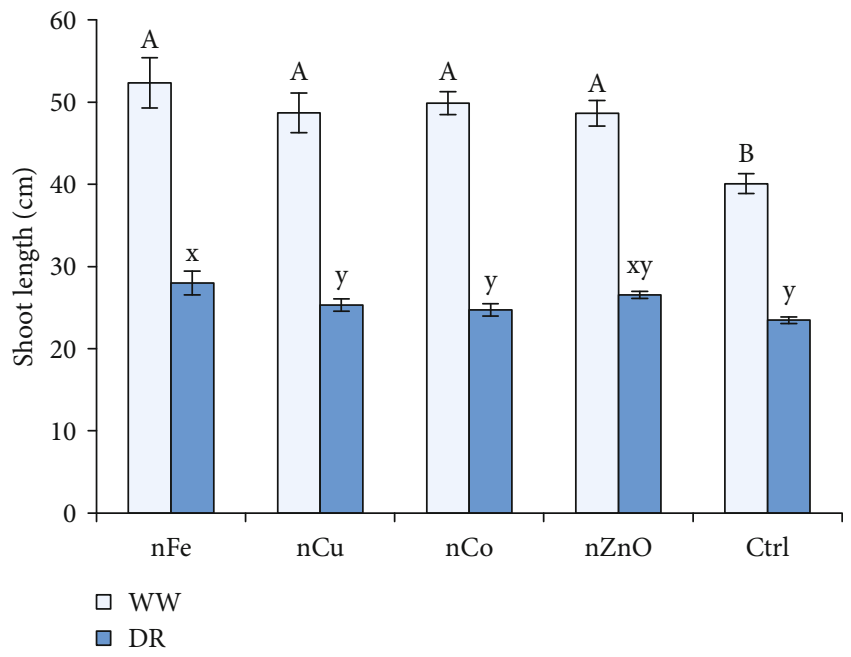

(c)

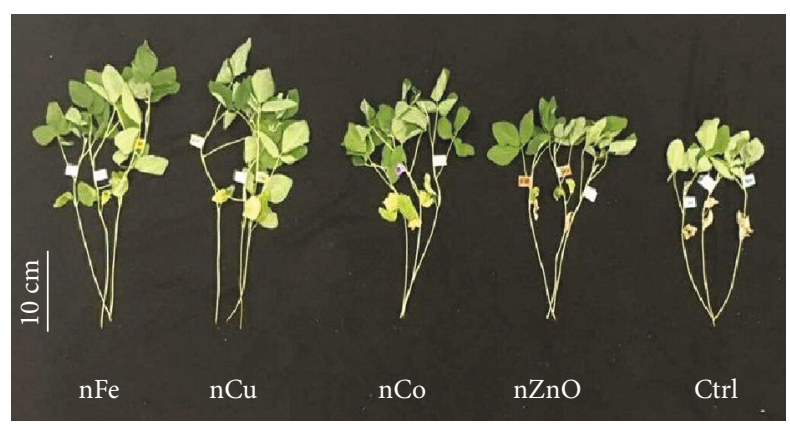

(b)

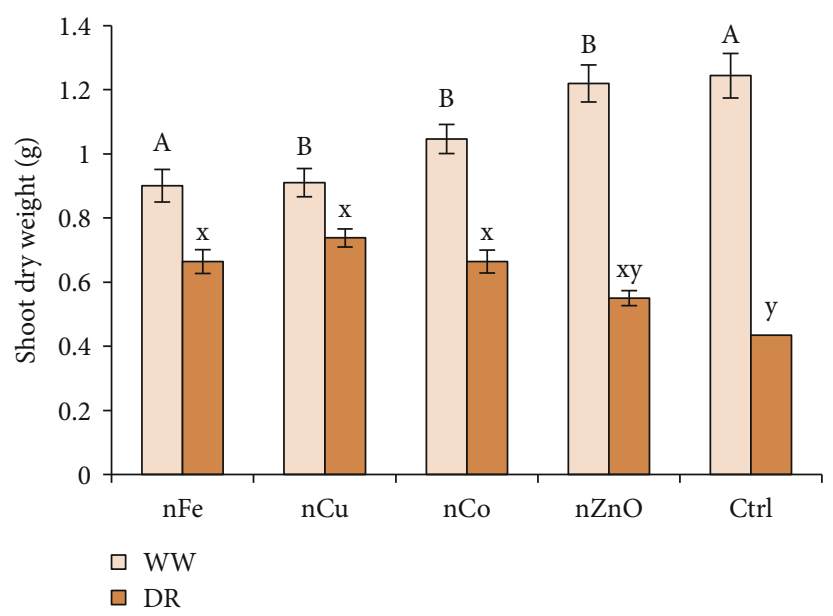

(d)

FIGURE 1: Shoot development of soybean seedlings under well-watered (WW) and induced drought (DR) conditions. (a) Shoot morphology of plants treated with $\mathrm{NPs}(\mathrm{nFe}, \mathrm{nCu}, \mathrm{nCo}$, and $\mathrm{nZnO}$ ) and control (Ctrl) plants under well-watered conditions. (b) Treatments identical to those in (a), but under induced drought conditions. (c) Shoot length. (d) Shoot dry weight of NP-treated and untreated plants under wellwatered or induced drought conditions. Error bars represent standard errors $(n=20)$. Different letters (A and B for well-watered and $\mathrm{x}$ and y for drought conditions) indicate significant differences within treatments, according to Tukey's test $(p<0.05)$.

2.4. Statistical Analysis. Data were statistically analyzed according to Student's $t$-test (significant differences with $p$ $<0.05$ are indicated by asterisks) or one-way analysis of variance (ANOVA) with significant differences among treatments at $p<0.05$ determined according to Tukey's test.

\section{Results}

3.1. Effect of NP Treatments on the Development of the Soybean DT26 Cultivar under Drought Conditions. The performance of NP-treated plants was analyzed under induced drought conditions to understand the possible benefits of NP application to the enhancement of soybean tolerance to a water deficit. The experiments were conducted under strictly monitored conditions to maintain soil moisture content (\% SMC) between 60 and 70\% in the control treatment groups and to gradually reduce SMC to $30-40 \%$ by the last day of drought treatment. The development of shoots and roots of these plants was analyzed after 15 days of induced drought treatment.

Shoot morphology analysis (Figures 1(a) and 1(b)) indicated that NP treatment promoted shoot development in both well-watered and drought-stressed plants at the vegeta- tive stage. Shoot length reached approximately $50 \mathrm{~cm}$ in NPtreated plants and only $40 \mathrm{~cm}$ in the NP-untreated control plants (Figure 1(c)), under well-watered conditions. NP treatment effectiveness was ranked as follows: $\mathrm{Fe} \mathrm{NP}>\mathrm{Co}$ $\mathrm{NP}>\mathrm{Cu} \mathrm{NP}>\mathrm{ZnO}$ NP. Furthermore, although plant height increased with NP treatment, shoot dry weight decreased from $1.2 \mathrm{~g}$ for controls to $0.9 \mathrm{~g}$ for Fe NP-treated plants under well-watered conditions, with the rest of NP treatments lying intermediate between these two extremes (Figure 1(d)). Conversely, although under drought stress conditions the differences in shoot length among $\mathrm{Co}, \mathrm{Cu}$, and $\mathrm{ZnO} \mathrm{NP}$ plants and controls were not statistically significant (Figure 1(c)), shoot dry weight of NP-treated plants was higher $(p<0.05$; Tukey's test) than that of untreated plants (Figure 1(d)).

Root growth was improved by NP treatment only under induced drought conditions. The roots of NP-treated plants were significantly longer ( $p<0.05$; Tukey's test) than those of control plants; particularly, Fe NP-treated plants produced the longest roots (average $80.1 \mathrm{~cm}$ ), followed by $\mathrm{Cu}, \mathrm{Co}$, and $\mathrm{ZnO} \mathrm{NP}$ plants with lengths between 75.4 and $78.1 \mathrm{~cm}$, compared to $67.6 \mathrm{~cm}$ in root length for control plants. Although root length increased, root dry weight was not significantly different between NP treatments and controls, neither in 


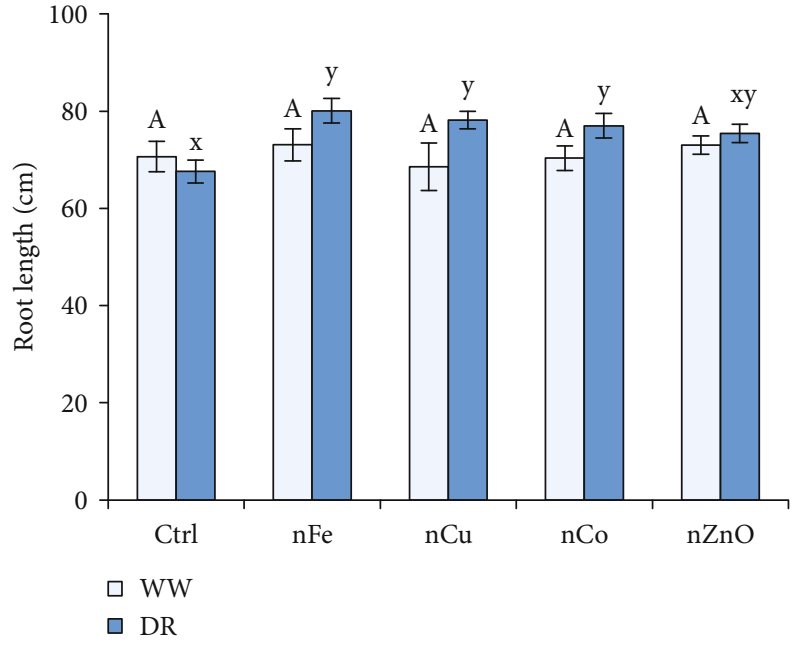

(a)

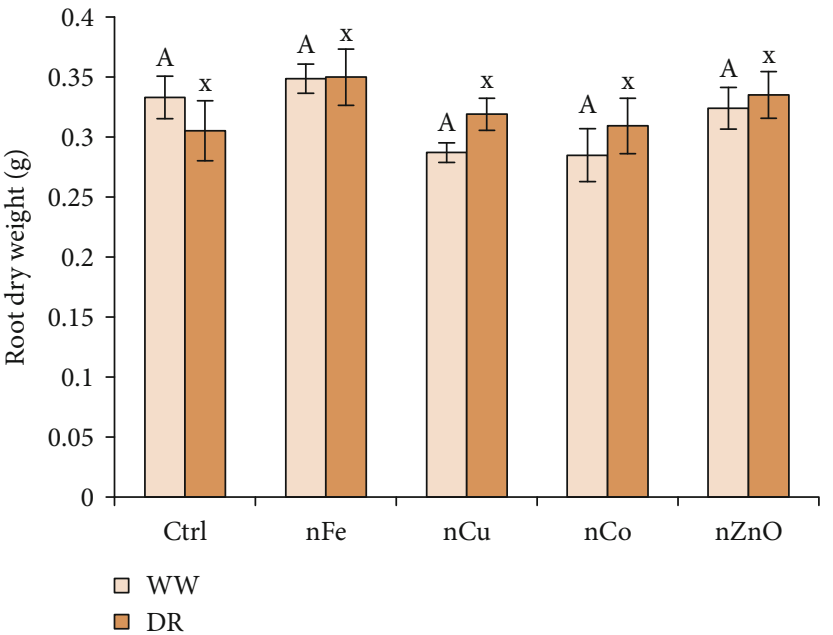

(b)

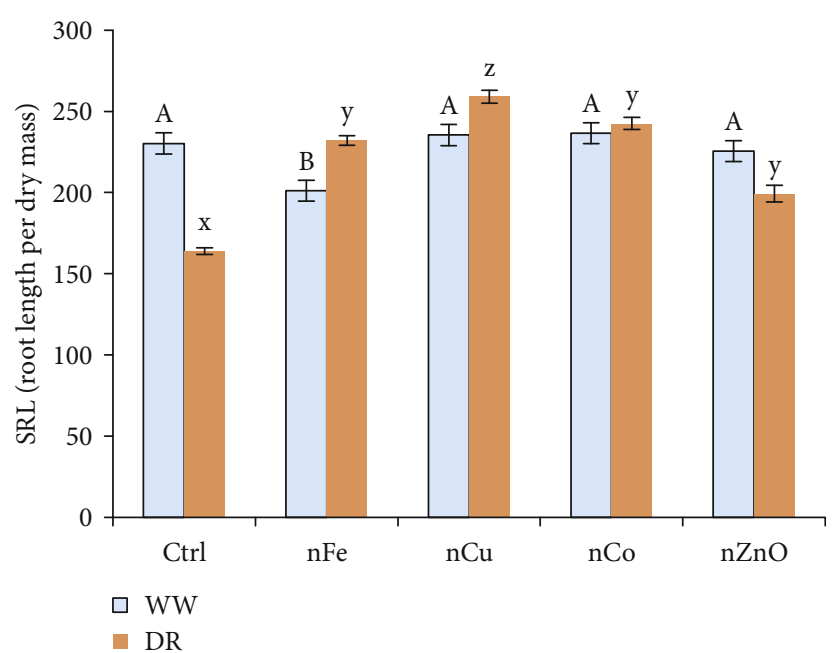

(c)

FIGURE 2: Root development of soybean seedlings under well-watered (WW) and induced drought (DR) conditions. (a) Root length of NPtreated ( $\mathrm{nFe}, \mathrm{nCu}, \mathrm{nCo}$, and $\mathrm{nZnO}$ ) and control (Ctrl) plants under well-watered and drought conditions. (b) Root dry weight for similar treatments as in (a). (c) SRL index under well-watered and induced drought conditions. Error bars represent standard errors $(n=20)$. Different letters (A, B, and C or $\mathrm{x}, \mathrm{y}$, and $\mathrm{z}$ ) indicate significant differences within treatments according to Tukey's test $(p<0.05)$.

well-watered nor in induced drought conditions (Figures 2(a) and 2(b)). Such root response resulted in greater specific root length (SRL) of NP-treated plants under induced drought conditions (Figure 2(c)).

3.2. Assessment of NP Treatment Effects on the Drought Tolerance Index. To assess the physical responses of NPtreated soybean plants under induced drought conditions, we measured relative water content (RWC), drought tolerance index (DTI), and biomass reduction rate, which are common physiological traits used to estimate plant drought tolerance. While RWC values provide information on plant tolerance level under drought conditions, DTI reveals more accurately plant capacity for drought tolerance. Thus, the higher the RWC and DTI values, the more drought tolerant the cultivar is.

Almost all NP-treated plant groups displayed RWC values similar to that of the untreated groups under well- watered conditions (Figure 3(a)). Conversely, all plant groups showed a similar trend of considerable reduction of RWC after the experimental drought period. However, under such induced drought conditions, $\mathrm{Fe}$ and $\mathrm{Cu}$ NP-treated plants maintained RWC at $71 \%$, which was significantly higher ( $p<0.05$; Tukey's test) than the RWC of control plants $(64 \%)$ (Figure 3(a)).

With respect to DTI, results shown in Figure 3(b) indicate that NP-treated plants exhibited higher DTI values compared to controls. Among NP-treated plants, Fe NP-treated plants showed the highest drought tolerance capacity, with a DTI value of 0.97 , which was sevenfold higher than the value for untreated plants, followed by $\mathrm{Co}, \mathrm{Cu}$, and $\mathrm{ZnO}$ NPs (Figure 3(b)). Significantly, although $\mathrm{Fe}$ and $\mathrm{Cu}$ NP-treated plants had a similar RWC, the DTI value of Fe NP-treated plants was twofold higher and significantly different from the DTI value of $\mathrm{Cu}$ NP-treated plants (0.41). 


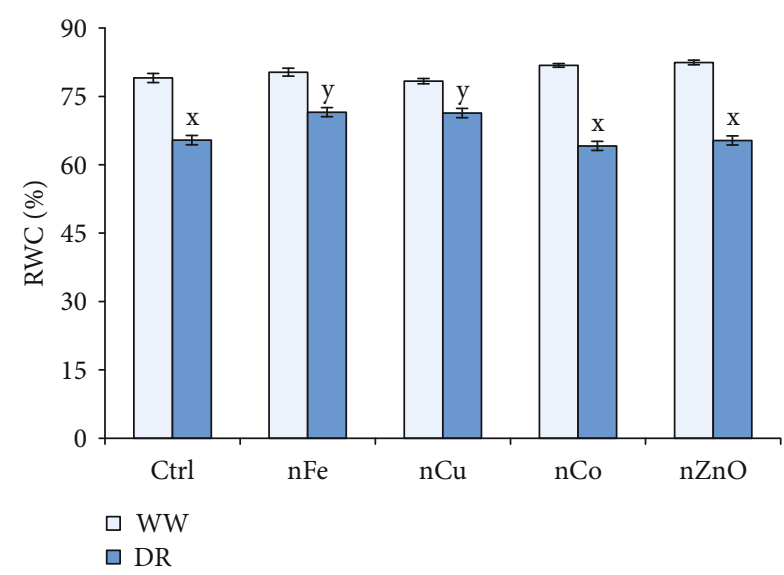

(a)

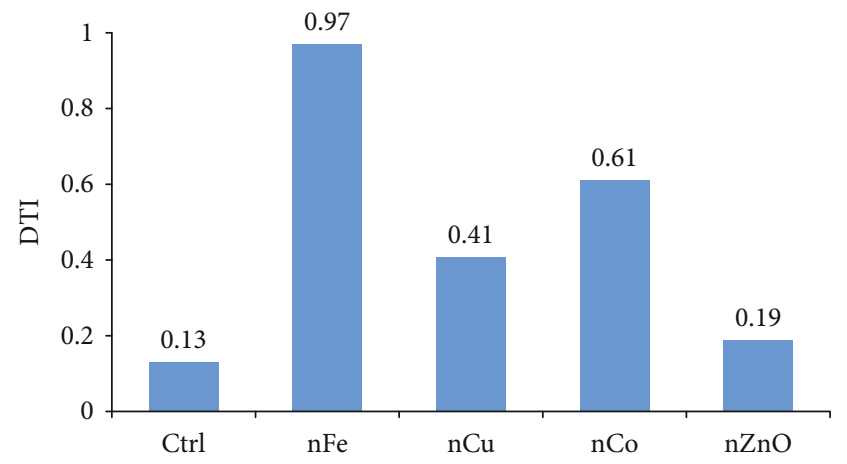

(b)

Figure 3: Analysis of the drought tolerance index in NP-treated plants under well-watered and drought-induced conditions. (a) Relative water content (RWC) of NP-treated $(\mathrm{nFe}, \mathrm{nCu}, \mathrm{nCo}$, and $\mathrm{nZnO})$ and control (Ctrl) soybean plants under well-watered (WW) and drought conditions (DR). Error bars represent standard errors $(n=20)$. Different letters $(\mathrm{x}, \mathrm{y})$ indicate significant differences within a treatment according to Tukey's test $(p<0.05)$. (b) Drought tolerance index (DTI) of NP-treated ( $\mathrm{nFe}, \mathrm{nCu}, \mathrm{nCo}$, and $\mathrm{nZnO})$ and control (Ctrl) soybean plants; DTI values were determined by the percentage of unwilted plants and plants showing recovery after $1,3,13$, and 15 days following drought exposure and rewatering $(n=30)$.

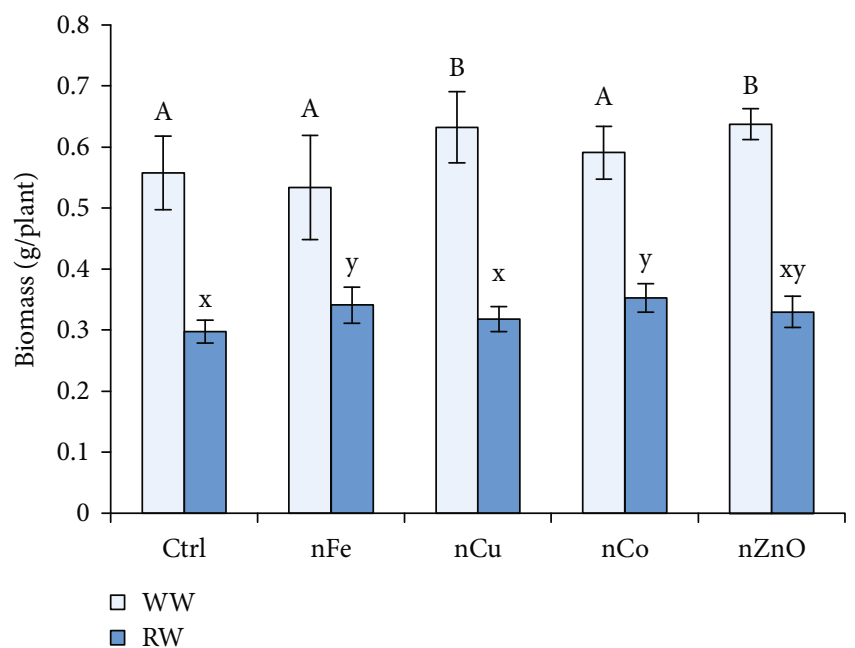

(a)

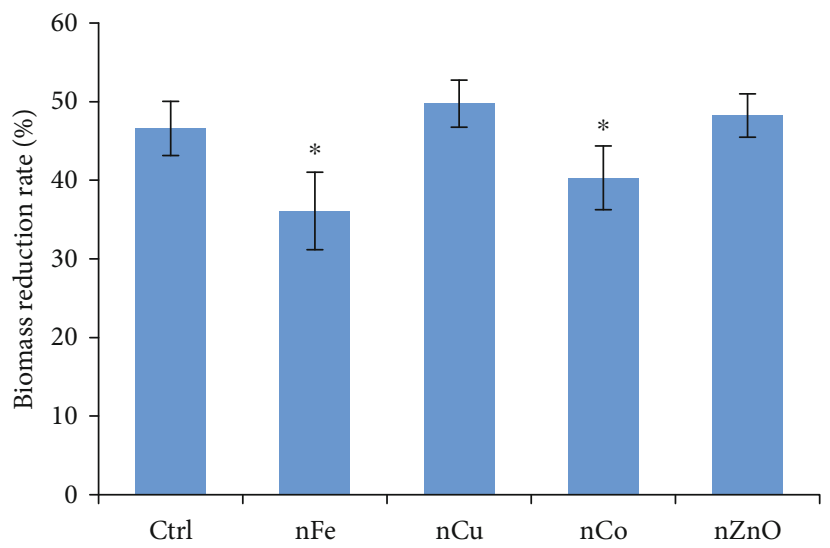

(b)

Figure 4: Biomass analysis of NP-treated and control plants under well-watered and drought-induced conditions. (a) Biomass (g/plant) of $\mathrm{NP}$-treated $(\mathrm{nFe}, \mathrm{nCu}, \mathrm{nCo}$, and $\mathrm{nZnO})$ and control (Ctrl) plants under well-watered conditions (WW) and drought/rewatering conditions (RW) (drought for 15 days and rewatering for seven days). Data represent the means and standard errors of a representative experiment ( $n=10$ plants/treatment). Different letters (A and B for well-watered and $\mathrm{x}$ and y for drought-induced conditions) indicate significant differences within treatments according to Tukey's test $(p<0.05)$. (b) Biomass reduction rate $(\%)$ of $\mathrm{NP}$-treated $(\mathrm{nFe}$, nCu, $\mathrm{nCo}$, and $\mathrm{nZnO}$ ) and control (Ctrl) plants under RW conditions relative to their respective well-watered controls. Data represent the means and standard errors of a representative experiment ( $n=7$ plants/treatment). Asterisks on top of the bars indicate statistically significant differences with ${ }^{*} p<0.05$ in all combinations, according to Tukey's test.

Plant biomass of $\mathrm{Cu}$ and $\mathrm{Zn}$ NP-treated plants $(0.63 \mathrm{~g})$ was higher than that of Fe or Co NP-treated plants $(0.53$ and $0.59 \mathrm{~g}$, respectively) under well-watered conditions. However, after the experimental drought period and subsequent rewatering, plant biomass of the former were 0.04$0.06 \mathrm{~g}$ lower than those of Fe or Co NP-treated plants or that of the control (Figure 4(a)). Therefore, the lowest biomass reduction rate was observed for Fe and Co NP-treated plants (36\% and $40 \%$, respectively), whereas it was $49 \%$ for $\mathrm{Cu} \mathrm{NP}$,
$48 \%$ for $\mathrm{Zn} \mathrm{NP}$, and $46 \%$ for the control treatment (Figure 4(b)). The calculated biomass reduction rates indicated that Fe and Co NP-treated plants maintained higher biomass under drought than untreated or $\mathrm{Cu}$ and $\mathrm{Zn} \mathrm{NP}$ treated plants.

3.3. Expression Analysis of Drought-Responsive Genes in NPTreated Soybean Plants. The expression of drought tolerance marker genes in leaves and roots of NP-treated and 

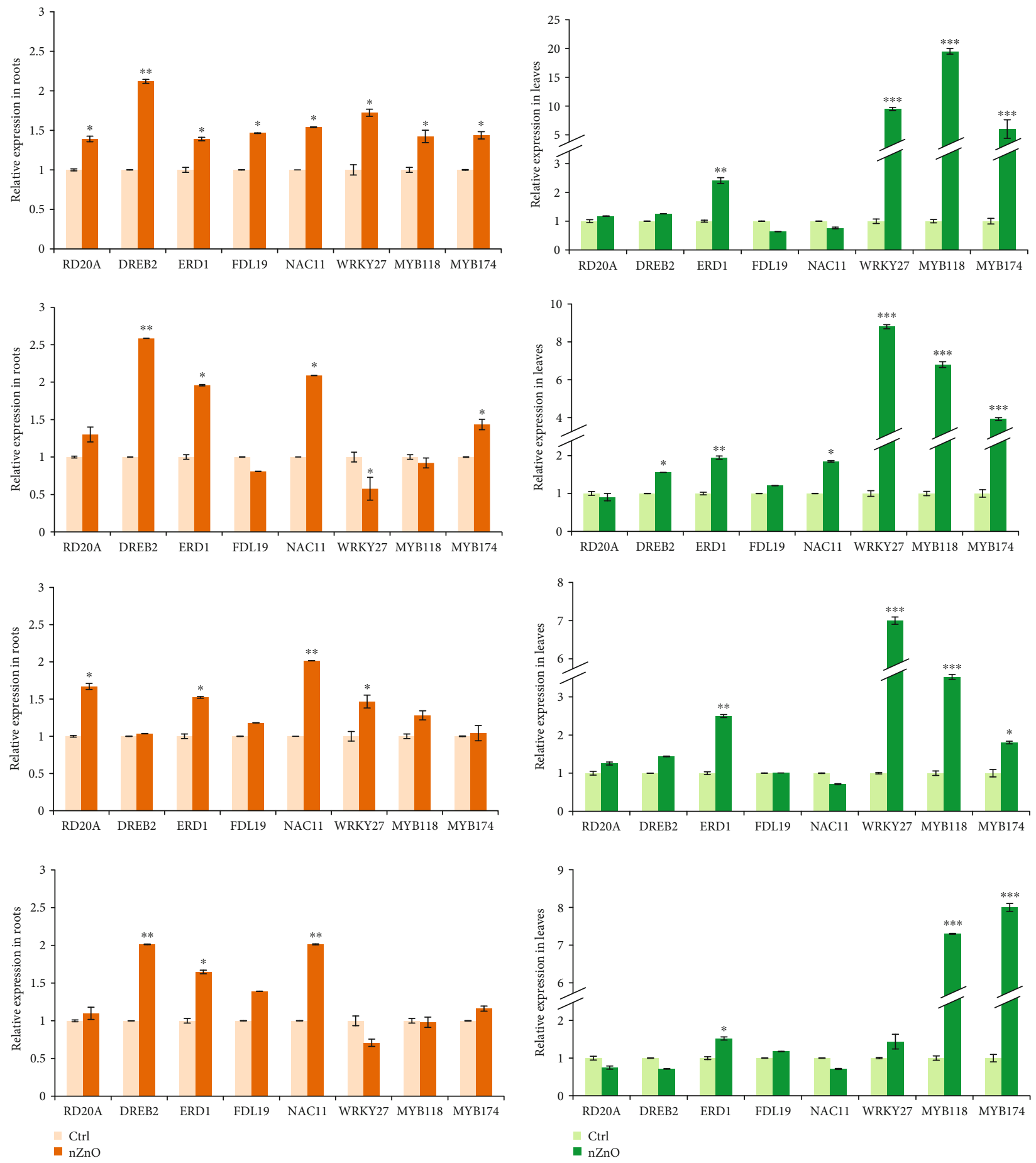

Figure 5: Expression patterns of GmRD20A, GmDREB2, GmERD1, GmFDL19, GmNAC11, GmWRKY27, GmMYB118, and GmMYB174 in root and leaf samples of $\mathrm{Fe}, \mathrm{Cu}, \mathrm{Co}$, and $\mathrm{ZnO} \mathrm{NP}$-treated soybean plants under drought conditions. Relative expression levels were normalized to a value of 1.0 in the NP-untreated plant samples (Ctrl). Asterisks above bars demonstrate statistically significant differences when compared with NP-untreated plants using Student's $t$-test with ${ }^{*} p<0.05,{ }^{* *} p<0.01$, or ${ }^{* * *} p<0.001$.

nontreated plants (controls) was analyzed using a qPCR method. The results shown in Figure 5 reveal that under induced drought conditions, selected drought tolerance marker genes (GmRD20A, GmDREB2, GmERD1, GmFDL19, GmNAC11, GmWRKY27, GmMYB118, and GmMYB174) were upregulated in roots or shoots of NP-treated plants, depending on the specific gene, the plant tissue, and the NP used. In Fe NP-treated plants, the expression of all genes analyzed was significantly higher in the roots $\left({ }^{*} p<0.05\right.$ or ${ }^{* *} p$ $<0.01$; $t$-test), while GmERD1, GmWRKY27, GmMYB118, and $G m M Y B 174$ showed significantly elevated expression in the leaves $\left({ }^{* *} p<0.01\right.$ or ${ }^{* * *} p<0.001 ; t$-test). Additionally, 
under drought-induced conditions, $\mathrm{Cu}$ NP treatment raised the transcript level for GmDREB2, GmERD1, GmNAC11, and $G m M Y B 174$ in roots and for GmDREB2, GmERD1, GmNAC11, GmWRKY27, GmMYB118, and GmMYB174 in leaves. In turn, Co NP treatment increased the expression level of GmRD20A, GmERD1, GmNAC11, and GmWRKY27 in roots and of GmERD1, GmWRKY27, GmMYB118, and GmMYB174 in leaves. Lastly, fewer marker genes were upregulated in both roots and leaves of $\mathrm{ZnO}$ NP-treated plants; these were GmDREB2, GmERD1, and GmNAC11 in roots and GmERD1, GmMYB118, and GmMYB174 in leaves.

The increase in the expression level of GmWRKY27, GmMYB118, and GmMYB174 in the leaves of all four NPtreated plant groups was the most remarkable change in gene expression observed, namely, 8- to 10-fold, 6- to 20-fold, and 2 - to 8-fold, respectively. Among all analyzed drought tolerance marker genes, GmERD1 was the only one that was upregulated in both roots and leaves, regardless of the NP used. Further, the expression of GmERD1 in leaves was highest (2.16-fold) in Fe NP-treated plants relative to untreated plants, followed by Co NP (2.03-fold), Cu NP (1.73-fold), and $\mathrm{ZnO} \mathrm{NP}$ (1.4-fold). Thus, our results clearly demonstrated that the expression of drought tolerance marker genes was effectively induced by $\mathrm{Fe}, \mathrm{Cu}, \mathrm{Co}$, and $\mathrm{ZnO}$ treatments.

\section{Discussion}

4.1. Metal-Based Nanoparticles Promote Plant Growth Responses to Water Stress Adaptation and Tolerance. The results described above clearly indicate that the NPs tested herein contributed to the increase in the tolerance of the soybean cultivar DT26 to drought, as reflected by our measurements of plant growth, RWC, DTI, and biomass reduction rate. NP-treated plants, especially with $\mathrm{Fe} \mathrm{NP}$, grew to a larger extent than control plants under both well-watered and induced drought conditions. Although the shoot length of NP-treated plants was similar to that of control plants, the dry weight of NP-treated plants markedly increased above the control level by the end of the drought period. This observation suggests continued photosynthetic activity in the leaves of NP-treated plants. It is also important to observe that root length and specific root length index (SRL, i.e., root length per unit dry mass) (Figure 2) of NP-treated plants increased compared to that of controls. They are among several root morphological characteristics associated with sustained productivity under drought conditions [41]. The higher SRL observed here suggests that NP-treated soybean plants might have supported root elongation under water stress, thereby permitting soybean plants to adapt/tolerate drought by accessing deeper soil water. Consistently, according to Comas et al. [42], small roots with larger SRL allow the plant to easily increase hydraulic conductivity by increasing the root surface area in contact with soil water and the soil volume that can be exploited for water.

The positive effects of metal-based NPs have been previously examined for drought tolerance in several other plant species. Thus, for example, Mozafari et al. [43] reported that the addition of iron NPs in an in vitro culture medium increased drought stress tolerance of Fragaria $\times$ ananassa
Duch. Similarly, the drought stress endurance of Ocimum basilicum improved upon application of $\mathrm{TiO}_{2} \mathrm{NPs}$ combined with gibberellin $\left(\mathrm{GA}_{3}\right)$ [44]. Furthermore, wheat productivity improved under water deficit conditions with $\mathrm{Cu}$ and Fe NP application through the increase in superoxide dismutase (SOD) activity and sugar content [45].

In addition to improving morphological characteristics, NP treatment-induced increase in drought tolerance was also reflected by RWC, which is the most appropriate measurement for plant water status in case of a cellular water deficit. In this study, Fe and Cu NPs seemingly supported cell water retention under water deficit conditions. Consistently with our results, $\mathrm{Cu}$ and $\mathrm{Zn}$ NPs induced an increase in RWC by $8 \%-10 \%$ in leaves of seedlings of two different wheat varieties exposed to drought [13]. In addition, such physiological parameters have been frequently used to screen for drought-tolerant genotypes in wheat [46], soybean [47], and finger millet [48].

Although the effects of $\mathrm{Fe}$ and $\mathrm{Cu}$ NPs on water retention were equal, Fe NP was found to be most effective in increasing drought tolerance in soybean (7-fold increase in DTI compared to the control), followed by Co NP (with 4-fold increase in DTI; Figure 3(b)). DTI is considered a more accurate index for drought tolerance assessment because it combines all tolerance parameters, i.e., recovery factor, tolerance index, and recovery index. In practice, DTI has been calculated as a single factor or a combination with others to estimate drought adaptability in maize [49], rice [50], sugarcane [51], and soybean [35]. Therefore, Fe and Co NP treatments might be applied to field-grown soybean under water deficit conditions. In plants, $\mathrm{Fe}$ and $\mathrm{Co}$ are indispensable elements and especially important for legume species such as soybean, to sustain plant development, nodule production, and nitrogen fixation $[6,52]$. The availability of these elements was necessary for enhancing or preparing plants to better tolerate drought stress [7, 53-56]. The effect of Fe NP has also been investigated in cowpea [57], but this is the first report related to NP-induced drought tolerance in soybean.

Shoot dry weight of NP-treated and untreated plants declined considerably under drought conditions compared with that of well-watered controls; however, it was higher in NP-treated plants than in untreated plants after rewatering. Consequently, the biomass reduction rate was lower in NP-treated plants than in controls, and Fe and Co NPtreated plants showed the lowest biomass reduction rate. Overall, our observations indicated that NPs, especially $\mathrm{Fe}$ NPs, had positive effects on soybean plants that enabled them to better adapt to drought conditions.

\subsection{Nanoparticles Induce Drought-Related Gene Expression.} In this study, the combination of physiological responses and expression analysis of representative genes may help understand how soybean plants and NPs interact at a molecular level to mitigate drought stress. The expression of the selected drought tolerance marker genes, GmRD20A, GmDREB2, GmERD1, GmFDL19, GmNAC11, GmWRKY27, $G m M Y B 118$, and $G m M Y B 174$, was found to be upregulated in roots or shoots (or both) of NP-treated plants under 
drought. The above genes have been employed as water deficit tolerance markers in several studies on Arabidopsis [17] and other species, including soybean $[18,58]$. Although these are drought-induced genes, according to Neves-Borges et al. [18] their expression pattern and induction levels were dependent on each specific gene and on drought experimental design. Among the tested NPs in our study, Fe NP was the most interesting because it was the only one to induce all marker genes in roots under drought stress conditions.

The increased expression of GmWRKY27, GmMYB118, and $G m M Y B 174$ in leaves proves that NP treatments do indeed trigger drought tolerance responses in plants. GmWRKY27 belongs to a superfamily of plant transcription factor genes, WRKY, and is involved in various physiological and developmental processes in plants, such as hormone signaling, catabolism, secondary biosynthesis, phosphate acquisition, lignin biosynthesis, seed germination, and stress responses [59]. The most important role of the WRKY transcription factor family is the regulation of plant defense and stress responses. WRKY proteins are known to regulate plant responses to a wide range of abiotic stress conditions including drought, flooding, heat, cold, heavy metal toxicity, low humidity, and osmotic, oxidative, salt, and UV stress [59]. According to Zhou et al. [60], GmWRKY27 was expressed at a low level in soybean roots and leaves under normal conditions but was highly induced by drought stress, salt stress, and abscisic acid (ABA) treatment. Notably, the expression of $G m W R K Y 27$ was found to increase strongly in the very early phase $(0.5-1 \mathrm{~h})$ of drought, salinity, or ABA treatment and decrease in the late phase $(12 \mathrm{~h})$ of treatment [60]. The high expression level of GmWRKY27 in the leaves of all NP-treated plants under drought conditions (Figure 5), but not in roots, suggests that NPs may be linked to the regulation of ABA biosynthesis and stomatal function under drought conditions. This is because plant cells are known to trigger ABA biosynthesis and accumulate ABA in the cytosol under stress conditions, leading to the regulation of stomatal opening and closure. This event is important to control water loss through transpiration under water deficit conditions [61]. On the contrary, WRKY27 might interact with other transcriptional activators to induce the expression of genes related to the ABA-dependent pathway, respond to water deficit stress, and trigger drought tolerance in soybean. The combined expression of GmWRKY27 and GmMYB174 is believed to suppress the expression of $G m N A C 29$, a negative effector, resulting in plants becoming tolerant to abiotic stress [23]. Overexpression of GmWRKY27 also produced transgenic soybean plants tolerant to salinity and water deficit stress [23].

GmMYB118 is one of the 156 GmMYB genes identified in soybean by Liao et al. [62]. In this study, all NPs promoted increased expression of GmMYB118 in leaves, but only Fe NP treatment enhanced its expression in both roots and leaves under drought conditions. Du et al. [24] postulated that the GmMYB118 transcription factor might improve tolerance to drought and salt stress by promoting the expression of stress-associated genes. The expression of GmMYB118 (synonym: GmMYB173) is also known to induce the salinity tolerance process by regulating flavonoid biosynthesis to reduce reactive oxygen species (ROS) content [63]. Therefore, GmMYB118 might be considered one of the most important transcription factors regulating soybean adaptation to drought conditions.

The upregulation of GmERD1 in both leaves and roots makes it the most sensitive gene under induced drought conditions in NP-treated plants. ERD1 functions in the ABAindependent pathway; it is a functional gene involved in a cascade of reactions acting directly in response to abiotic stress $[58,64]$. It was shown to be strongly expressed in a drought-sensitive soybean cultivar under water deficit conditions, but not in the tolerant counterpart $[18,58]$. According to Neves-Borges et al. [18], ERD1 plays an important role at an earlier stage in the drought response pathway. Therefore, the increased expression of GmERD1 in leaves and roots of NP-treated plants suggests that ERD1 has a function in common responses at an early stage of an adverse signal response pathway in soybean. Neves-Borges et al. [18] considered that the upregulation of GmERD1 might be important for plants lacking traits needed for plant adaptation to drought, which might explain the drought stress responses of the nondrought-tolerant DT26 soybean cultivar, through the enhancement of GmERD1 expression by NP treatment.

In contrast with ERD1, RD20A is considered part of the ABA-dependent pathway [64]. The study of Neves-Borges et al. [18] on soybean indicated that the expression of $R D 20 A$ dramatically increased in roots of drought-stressed plants growing in a potted soil system (Psy), but not in plants abruptly subjected to drought, as compared to plants growing in a hydroponic system (Hsy) that can be made to change suddenly. In this study, water stress treatment was carried out in potted soil in which soil moisture content was controlled by a method similar to those in the Psy system, which allowed plants to gradually adapt to water shortage. In this system, the expression of RD20A in our experiments was remarkably enhanced only in roots of Fe or Co NP-treated plants. The results indicated that Fe and Co NPs had a strong effect on the expression of drought marker genes in roots, which might explain the morphological changes of roots and DTI values in Fe and Co NP-treated plants.

In addition to $R D 20 A$, the expression of GmNAC11 increased in roots of all NP treatments, while that of DREB2 increased in roots of the $\mathrm{Fe}, \mathrm{Cu}$, and $\mathrm{ZnO} \mathrm{NP}$-treated plants. NAC transcription factors are also known to activate gene expression via stimulating other transcriptional activators such as DREB2 or ERD1 [64]. According to Hao et al. [25], GmNAC11 expression was induced by adverse environmental factors such as salinity and dehydration; transgenic plants were resistant to salinity following overexpression of GmNAC11. Hao et al. [25] also indicated that GmNAC11 had a role in transcription initiation in combination with DREB1A. The increased expression of GmNAC11 and GmDREB2 in the roots of most NP-treated plants strengthens the hypothesis that NPs in our study relate to the expression of genes involved in the ABA-independent pathway. Overall, metal-based NPs can affect the expression of drought-inducible genes through both ABA-dependent and ABA-independent pathways under drought conditions, subject to particular NP type and specific plant tissues. 


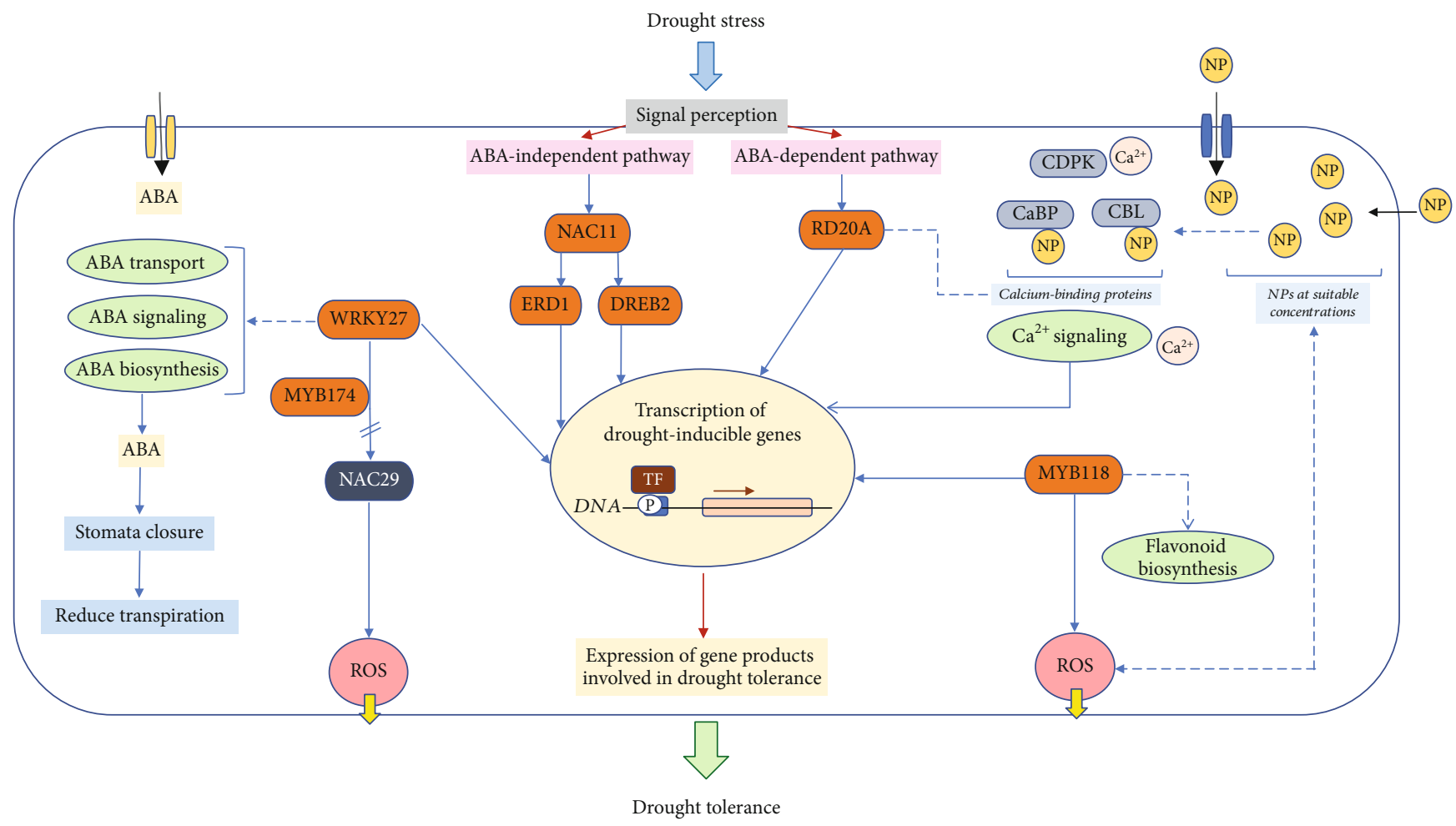

Figure 6: Illustration of the possible drought-responsive mechanism in soybean cells triggered by nanoparticles. CaBP, CDPK, CBL: calciumbinding proteins; NP: nanoparticle; ROS: reactive oxygen species; TF: transcription factor; P: promoter. Solid arrows indicate characterized pathways. Dotted arrows and lines indicate possible functions or hypothesized pathways.

It is well known that the response of plant cells to environmental stress is a complex process involving a network of signaling pathways [64]. Although precise modes of action of NPs in such conditions have been investigated, several hypotheses have emerged. The hypothesis that best matched our findings is that of Khan et al. [15], in which NPs are considered to act as $\mathrm{Ca}^{2+}$ or signaling molecules in the cytoplasm, identified by the calcium-binding protein (CaBP) complex or nanoparticle-specific proteins once inside plant cells. Consequently, a series of intracellular signaling pathways are induced and the expression of associated genes is activated, leading to enhanced plant tolerance responses to adverse conditions. In Arabidopsis, the expression of the $R D 20$ gene was induced by drought and salinity conditions or by ABA treatment [65]. Further analysis indicated that the RD20 protein harbors a conservative and specific region for calcium ion binding (EF-hand) [65]. The enhancement of GmRD20A expression in this study, especially in Fe and Co NP-treated plants, clearly supports the proposed hypothesis that nanoparticles may participate in inducing $\mathrm{Ca}^{2+}$ binding protein expression. In addition, NPs are also believed to play a role in ROS scavenging by simulating antioxidative enzyme activities. Recently, Sun et al. [66] provided strong evidence for the above theory, in which the expression of $\mathrm{Fe} / \mathrm{Mn} \mathrm{SOD}, \mathrm{Cu} / \mathrm{Zn} \mathrm{SOD}, \mathrm{APX}$, and CAT was significantly enhanced in $\mathrm{ZnO}$ NP-treated plants under drought. Based on our own findings and other reported studies, a possible mechanism of NP-mediated induction of drought tolerance of plants is illustrated in Figure 6.
As shown, NPs enter the cells by penetrating or by transport through specific channels in the cell membrane. They may act as stress signaling molecules and induce expression of drought-responsive genes (including regulatory factors) leading to defense system activation and tolerance to stress. NPs are proposed to mimic $\mathrm{Ca}^{2+}$ ions and bind with calcium-binding proteins (CaBP, CDPK, and CBL) [15]. The activated CaBP directly binds to the promoters of drought-responsive genes in the $\mathrm{Ca}^{2+}$ signaling pathway and then triggers the expression of downstream droughtrelated genes. Additionally, a suitable amount of NPs can maintain an appropriate level of ROS to stimulate the ROS signaling network for activation of the plant defense system under drought conditions. In this study, seven regulatory genes-GmWRKY27, GmMYB174, GmMYB118, GmERD1, GmDREB2, GmRD20A, and GmNAC11 (shown in orange boxes)-were highly expressed in NP-treated soybean plants under induced drought conditions. GmWRKY27 and GmMYB174 factors cooperatively inhibit the expression of GmNAC29, a transcriptional repressor, that leads to reduced ROS levels [23]. The high expression of GmWRKY27 in leaves may regulate $\mathrm{ABA}$ biosynthesis and the $\mathrm{ABA}$ signaling pathway. As previously reported, ERD1 and DREB2 belong to the ABA-independent pathways, while RD20A plays roles in the ABA-dependent pathway [64]. The NAC11 factor may activate gene expression by stimulating other transcriptional activators such as ERD1 or DREB2 in ABA-independent pathways [64]. In Arabidopsis, the RD20 protein contains a conserved EF-hand $\mathrm{Ca}^{2+}$-binding domain that has been 
shown to bind to $\mathrm{Ca}^{2+}[65]$, suggesting that GmRD20A may relate to the calcium signaling pathway and interact with $\mathrm{Ca}^{2+}$ ions or NPs. The GmMYB118 factor might improve drought tolerance by promoting the expression of droughtrelated genes, reducing the ROS level, and regulating osmotic substances and flavonoid biosynthesis [24, 63].

\section{Conclusions}

The results of the present study demonstrated that $\mathrm{Fe}, \mathrm{Cu}$, $\mathrm{Co}$, and $\mathrm{ZnO} \mathrm{NP}$ treatments effectively helped soybean plants at an early vegetative stage to adapt to drought stress. In addition to the improvement of shoot and root morphology and drought tolerance indices, the expressions of the tested drought tolerance marker genes were significantly enhanced under water deficit conditions in NP-treated plants, compared with untreated control plants. Therefore, metal-based NPs might promote plant tolerance to drought stress through the induction of drought-related gene expression. The usefulness of NP treatment for coping with drought stress in soybean and other plant species of commercial interest at other plant development stages warrants further research.

\section{Data Availability}

The data used to support the findings reported herein are available from the corresponding author upon request.

\section{Conflicts of Interest}

The authors declare no conflict of interest.

\section{Acknowledgments}

We gratefully thank A/Prof. Dr. N.P. Thao and her research group (School of Biotechnology, International University, Vietnam National University HCMC) for the technical support in drought experiments. We would also like to thank Prof. Rony Swennen, Dr. Jassmine Zorrilla, and Mrs. Hien Do (Laboratory of Tropical Crop Improvement, Department of Biosystems, Faculty of Bioscience Engineering, KU Leuven, Belgium) for their support in the qPCR experiments and analysis. Finally, we thank Editage (https://www.editage .com) for English language editing. This research was funded by the key project "Application of Nanotechnology in Agriculture” (code: VAST.TĐ.NANO-NN/15-18) financed by the Vietnam Academy of Science and Technology.

\section{References}

[1] M. C. Pagano and M. Miransari, "The importance of soybean production worldwide," Abiotic and Biotic Stresses in Soybean Production, vol. 1, pp. 1-26, 2016.

[2] B. Valliyodan, H. Ye, L. Song, M. K. Murphy, J. G. Shannon, and H. T. Nguyen, "Genetic diversity and genomic strategies for improving drought and waterlogging tolerance in soybeans," Journal of Experimental Botany, vol. 68, no. 8, pp. erw433-er1849, 2016.

[3] E. A. Waraich, R. Ahmad, M. Saifullah, Y. Ashraf, and M. Ehsanullah, "Role of Mineral Nutrition in Alleviation of
Drought Stress in Plants," Australian Journal of Crop Science, vol. 5, no. 6, pp. 764-777, 2011.

[4] F. Monjezi, F. Vazin, and M. Hassanzadehdelouei, "Effects of iron and zinc spray on yield and yield components of wheat (Triticum aestivum L.) in drought stress," Cercetari agronomice in Moldova, vol. 46, no. 1, pp. 23-32, 2013.

[5] M. Pourghola, N. Nemati, and M. Oveysi, "Effect of zinc and iron under the influence of drought on yield and yield components of rapeseed (Brassica napus)," Annals of Biological Research, vol. 4, no. 4, pp. 186-189, 2013.

[6] D. K. Tripathi, S. Singh, S. Gaur et al., "Acquisition and homeostasis of iron in higher plants and their probable role in abiotic stress tolerance," Frontiers in Environmental Science, vol. 5, 2018.

[7] M. Y. Ashraf, K. Mahmood, M. Ashraf, J. Akhter, and F. Hussain, "Optimal supply of micronutrients improves drought tolerance in legumes," Crop Production for Agricultural Improvement, pp. 637-657, 2012.

[8] C. A. Jaleel, Z. Changxing, K. Jayakumar, and M. Iqbal, "Low Concentration of Cobalt Increases Growth, Biochemical Constituents, Mineral Status and Yield in Zea Mays," Journal of Scientific Research, vol. 1, no. 1, pp. 128-137, 2008.

[9] R. Saxena, R. S. Tomar, and M. Kumar, "Exploring nanobiotechnology to mitigate abiotic stress in crop plants," Journal of Pharmaceutical Sciences and Research, vol. 8, no. 9, pp. 974-980, 2016.

[10] M. Sedghi, H. Mitra, and T. Sahar, "Effect of nano zinc oxide on the germination of soybean seeds under drought stress," Annals of West University of Timisoara: Series of Biology, vol. 16, no. 2, pp. 73-78, 2013.

[11] C. O. Dimkpa, U. Singh, P. S. Bindraban, W. H. Elmer, J. L. Gardea-Torresdey, and J. C. White, "Zinc oxide nanoparticles alleviate drought-induced alterations in sorghum performance, nutrient acquisition, and grain fortification," Science of The Total Environment, vol. 688, pp. 926-934, 2019.

[12] M. N. Helaly, M. A. El-Metwally, H. El-Hoseiny, S. A. Omar, and N. I. El-Sheery, "Effect of nanoparticles on biological contamination of 'in vitro' cultures and organogenic regeneration of banana," Australian Journal of Crop Science, vol. 8, no. 4, pp. 612-624, 2014

[13] N. Taran, V. Storozhenko, N. Svietlova, L. Batsmanova, V. Shvartau, and M. Kovalenko, "Effect of zinc and copper nanoparticles on drought resistance of wheat seedlings," Nanoscale Research Letters, vol. 12, no. 1, p. 60, 2017.

[14] J.-H. Kim, Y. Oh, H. Yoon, I. Hwang, and Y.-S. Chang, "Iron nanoparticle-induced activation of plasma membrane $\mathrm{H}^{+}$ATPase promotes stomatal opening in Arabidopsis thaliana," Environmental Science \& Technology, vol. 49, no. 2, pp. 1113-1119, 2015.

[15] M. N. Khan, M. Mobin, Z. K. Abbas, K. A. AlMutairi, and Z. H. Siddiqui, "Role of nanomaterials in plants under challenging environments," Plant Physiology and Biochemistry, vol. 110, pp. 194-209, 2017.

[16] G. Thapa, M. Dey, L. Sahoo, and S. K. Panda, "An insight into the drought stress induced alterations in plants," Biologia Plantarum, vol. 55, no. 4, pp. 603-613, 2011.

[17] G. T. Huang, S. L. Ma, L. P. Bai et al., "Signal transduction during cold, salt, and drought stresses in plants," Molecular Biology Reports, vol. 39, no. 2, pp. 969-987, 2012.

[18] A. C. Neves-Borges, F. Guimarães-Dias, F. Cruz et al., "Expression pattern of drought stress marker genes in soybean roots 
under two water deficit systems," Genetics and Molecular Biology, vol. 35, 1 suppl 1, pp. 212-221, 2012.

[19] D. Golldack, I. Lüking, and O. Yang, "Plant tolerance to drought and salinity: stress regulating transcription factors and their functional significance in the cellular transcriptional network," Plant Cell Reports, vol. 30, no. 8, pp. 1383-1391, 2011.

[20] J. Jin, H. Zhang, L. Kong, G. Gao, and J. Luo, "PlantTFDB 3.0: a portal for the functional and evolutionary study of plant transcription factors," Nucleic Acids Research, vol. 42, no. D1, pp. D1182-D1187, 2013.

[21] Y. Li, Q. Chen, H. Nan et al., "Over-expression of GmFDL19 enhances tolerance to drought and salt stresses in soybean," PLOS ONE, vol. 12, no. 6, article 0179554, 2017.

[22] M. Chen, Q.-Y. Wang, X.-G. Cheng et al., "GmDREB2, a soybean DRE-binding transcription factor, conferred drought and high-salt tolerance in transgenic plants," Biochemical and Biophysical Research Communications, vol. 353, no. 2, pp. 299305, 2007.

[23] F. Wang, H. W. Chen, Q. T. Li et al., "GmWRKY 27 interacts with GmMYB 174 to reduce expression of GmNAC 29 for stress tolerance in soybean plants," The Plant Journal, vol. 83, no. 2, pp. 224-236, 2015.

[24] Y.-T. Du, M.-J. Zhao, C.-T. Wang et al., "Identification and characterization of GmMYB118 responses to drought and salt stress," BMC Plant Biology, vol. 18, no. 1, p. 320, 2018.

[25] Y. J. Hao, W. Wei, Q. X. Song et al., "Soybean NAC transcription factors promote abiotic stress tolerance and lateral root formation in transgenic plants," The Plant Journal, vol. 68, no. 2, pp. 302-313, 2011.

[26] M. V. J. Da Costa and P. K. Sharma, "Effect of copper oxide nanoparticles on growth, morphology, photosynthesis, and antioxidant response in Oryza sativa," Photosynthetica, vol. 54, no. 1, pp. 110-119, 2016.

[27] M. Askary, S. M. Talebi, F. Amini, and A. D. B. Bangan, "Effects of iron nanoparticles on Mentha piperita L. under salinity stress," Biologija, vol. 63, no. 1, pp. 65-75, 2017.

[28] R. C. Choudhary, R. V. Kumaraswamy, S. Kumari et al., "Cuchitosan nanoparticle boost defense responses and plant growth in maize (Zea mays L.)," Scientific Reports, vol. 7, no. 1, article 9754, 2017.

[29] M. Jalali, F. Ghanati, A. M. Modarres-Sanavi, and A. H. Khoshgoftarmanesh, "Physiological effects of repeated foliar application of magnetite nanoparticles on maize plants," Journal of Agronomy and Crop Science, vol. 203, no. 6, pp. 593-602, 2017.

[30] A. A. H. A. Latef, M. F. A. Alhmad, and K. E. Abdelfattah, "The possible roles of priming with $\mathrm{ZnO}$ nanoparticles in mitigation of salinity stress in lupine (Lupinus termis) plants," Journal of Plant Growth Regulation, vol. 36, no. 1, pp. 60-70, 2017.

[31] M. Iqbal, N. I. Raja, Z.-U.-R. Mashwani, M. Hussain, M. Ejaz, and F. Yasmeen, "Effect of silver nanoparticles on growth of wheat under heat stress," Iranian Journal of Science and Technology, Transactions A: Science, vol. 43, no. 2, pp. 387-395, 2019.

[32] F. Pérez-Labrada, E. R. López-Vargas, H. Ortega-Ortiz, G. Cadenas-Pliego, A. Benavides-Mendoza, and A. JuárezMaldonado, "Responses of tomato plants under saline stress to foliar application of copper nanoparticles," Plants, vol. 8, no. 6, 2019.
[33] Q. B. Ngo, T. H. Dao, H. C. Nguyen et al., "Effects of nanocrystalline powders (Fe, Co and $\mathrm{Cu}$ ) on the germination, growth, crop yield and product quality of soybean (Vietnamese species DT-51)," Advances in Natural Sciences: Nanoscience and Nanotechnology, vol. 5, no. 1, article 015016, 2014.

[34] P. T. Hoe, N. C. Mai, L. Q. Lien et al., "Germination responses of soybean seeds under $\mathrm{Fe}, \mathrm{ZnO}$, cu and co nanoparticle treatments," International Journal of Agriculture and Biology, vol. 20, no. 7, pp. 1562-1568, 2018.

[35] N. B. A. Thu, Q. T. Nguyen, X. L. T. Hoang, N. P. Thao, and L. S. P. Tran, "Evaluation of drought tolerance of the Vietnamese soybean cultivars provides potential resources for soybean production and genetic engineering," BioMed Research International, vol. 2014, Article ID 809736, 9 pages, 2014.

[36] M. M. Hossain, X. Liu, X. Qi, H. M. Lam, and J. Zhang, "Differences between soybean genotypes in physiological response to sequential soil drying and rewetting," The Crop Journal, vol. 2, no. 6, pp. 366-380, 2014.

[37] K. H. Nguyen, M. G. Mostofa, W. Li et al., "The soybean transcription factor GmNAC085 enhances drought tolerance in Arabidopsis," Environmental and Experimental Botany, vol. 151, pp. 12-20, 2018.

[38] D. T. Le, D. L. Aldrich, B. Valliyodan et al., "Evaluation of candidate reference genes for normalization of quantitative RTPCR in soybean tissues under various abiotic stress conditions," PLOS ONE, vol. 7, no. 9, article e46487, 2012.

[39] N. Thao, N. Thu, X. Hoang, C. Ha, and L.-S. Tran, "Differential Expression Analysis of a Subset of Drought-Responsive GmNAC Genes in Two Soybean Cultivars Differing in Drought Tolerance," International Journal of Molecular Sciences, vol. 14, no. 12, pp. 23828-23841, 2013.

[40] K. J. Livak and T. D. Schmittgen, "Analysis of Relative Gene Expression Data Using Real-Time Quantitative PCR and the $2^{-\Delta \Delta \mathrm{C}}$ Tethod," Methods, vol. 25, no. 4, pp. 402-408, 2001.

[41] A. H. Fitter, "Plant Roots," in Characteristics and functions of root systems, pp. 21-50, The Hidden Half, New York, 3rd edition, 2002.

[42] L. H. Comas, S. R. Becker, V. M. V. Cruz, P. F. Byrne, and D. A. Dierig, "Root traits contributing to plant productivity under drought," Frontiers in Plant Science, vol. 4, 2013.

[43] A.-a. Mozafari, A. G. Asl, and N. Ghaderi, "Grape response to salinity stress and role of iron nanoparticle and potassium silicate to mitigate salt induced damage under in vitro conditions," Physiology and Molecular Biology of Plants, vol. 24, no. 1, pp. 25-35, 2018.

[44] H. Kiapour, P. Moaveni, D. Habibi, and B. Sani, "Evaluation of the application of gibbrellic acid and titanium dioxide nanoparticles under drought stress on some traits of basil (Ocimum basilicum L.)," International Journal Of Agronomy And Agricultural Research, vol. 6, pp. 138-150, 2015.

[45] F. Yasmeen, N. I. Raja, A. Razzaq, and S. Komatsu, "Proteomic and physiological analyses of wheat seeds exposed to copper and iron nanoparticles," Biochimica et Biophysica Acta (BBA) - Proteins and Proteomics, vol. 1865, no. 1, pp. 28-42, 2017.

[46] A. K. Yadav, A. J. Carroll, G. M. Estavillo, G. J. Rebetzke, and B. J. Pogson, "Wheat drought tolerance in the field is predicted by amino acid responses to glasshouse-imposed drought," Journal of Experimental Botany, vol. 70, no. 18, pp. 49314948, 2019.

[47] J. Chowdhury, M. Karim, Q. Khaliq, A. Ahmed, and A. M. Mondol, "Effect of drought stress on water relation traits of 
four soybean genotypes," SAARC Journal of Agriculture, vol. 15, no. 2, pp. 163-175, 2018.

[48] A. Mukami, A. Ngetich, C. Mweu, R. O. Oduor, M. Muthangya, and W. M. Mbinda, "Differential characterization of physiological and biochemical responses during drought stress in finger millet varieties," Physiology and Molecular Biology of Plants, vol. 25, no. 4, pp. 837-846, 2019.

[49] A. M. N. Al-Naggar, M. M. Shafik, and M. O. A. Elsheikh, "Putative mechanisms of drought tolerance in maize (Zea mays L.) via root system architecture traits," Annual Research \& Review in Biology, vol. 32, no. 2, pp. 1-19, 2019.

[50] S. Pimratch, T. Mungkunkamchao, and T. Kesmala, "Variations in morphological traits, agronomic traits and traits related to drought tolerance in indigenous Thai rice varieties," ScienceAsia, vol. 45, no. 2, pp. 99-108, 2019.

[51] T. H. Dinh, H. Takaragawa, K. Watanabe, M. Nakabaru, and Y. Kawamitsu, "Leaf photosynthesis response to change of soil moisture content in sugarcane," Sugar Tech, vol. 21, no. 6, pp. 949-958, 2019.

[52] I. Bonilla and L. Bolaños, "Mineral nutrition for legumerhizobia symbiosis: B, Ca, N, P, S, K, Fe, Mo, Co, and Ni: a review," Sustainable Agriculture Reviews, vol. 1, pp. 253-274, 2009.

[53] E. Ebrahimian and A. Bybordi, "Effect of iron foliar fertilization on growth, seed and oil yield of sunflower grown under different irrigation regimes," Middle East Journal of Scientific Research, vol. 9, no. 5, pp. 621-627, 2011.

[54] E. Elanz, R. Mohsen, and B. Ahmed, "Influence of salt stress on cations concentration quality and quantity of sunflower cultivars," Journal of Food, Agriculture and Environment, vol. 2, pp. 469-476, 2011.

[55] A. Pirzad and F. Shokrani, "Effects of Iron Application on Growth Characters and Flower Yield of Calendula officinalis L. Under Water Stress," World Applied Sciences Journal, vol. 18, no. 9, pp. 1203-1208, 2012.

[56] D. Z. Farinaz, R. Arash, and H. Amir, "Evaluation the effect of water stress and foliar application of Fe nanoparticles on yield, yield components and oil percentage of safflower (Carthamus tinctorious L.)," International Journal of Advanced Biological and Biomedical Research, vol. 2, no. 4, pp. 1150-1159, 2014.

[57] R. M. Afshar, H. Hadi, and A. Pirzad, "Effect of nano-iron foliar application on qualitative and quantitative characteristics of cowpea, under end season drought stress," International Research Journal Of Applied And Basic Sciences, vol. 3, no. 8, pp. 1709-1717, 2012.

[58] R. Stolf-Moreira, E. G. M. Lemos, L. Carareto-Alves et al., "Transcriptional profiles of roots of different soybean genotypes subjected to drought stress," Plant Molecular Biology Reporter, vol. 29, no. 1, pp. 19-34, 2011.

[59] P. J. Rushton, I. E. Somssich, P. Ringler, and Q. J. Shen, "WRKY transcription factors," Trends in Plant Science, vol. 15, no. 5, pp. 247-258, 2010.

[60] Q.-Y. Zhou, A.-G. Tian, H.-F. Zou et al., "Soybean WRKYtype transcription factor genes, GmWRKY13, GmWRKY21, and GmWRKY54, confer differential tolerance to abiotic stresses in transgenic Arabidopsis plants," Plant Biotechnology Journal, vol. 6, no. 5, pp. 486-503, 2008.

[61] C. Lim, W. Baek, J. Jung, J.-H. Kim, and S. Lee, "Function of ABA in stomatal defense against biotic and drought stresses," International Journal of Molecular Sciences, vol. 16, no. 12, pp. 15251-15270, 2015.
[62] Y. Liao, H. F. Zou, H. W. Wang et al., "Soybean GmMYB76, GmMYB92, and GmMYB177 genes confer stress tolerance in transgenic Arabidopsis plants," Cell Research, vol. 18, no. 10, pp. 1047-1060, 2008.

[63] E. Pi, C. Zhu, W. Fan et al., "Quantitative phosphoproteomic and metabolomic analyses reveal GmMYB173 optimizes flavonoid metabolism in soybean under salt stress," Molecular \& Cellular Proteomics, vol. 17, no. 6, pp. 1209-1224, 2018.

[64] K. Shinozaki and K. Yamaguchi-Shinozaki, "Gene networks involved in drought stress response and tolerance," Journal of Experimental Botany, vol. 58, no. 2, pp. 221-227, 2006.

[65] S. Takahashi, T. Katagiri, K. Yamaguchi-Shinozaki, and K. Shinozaki, "An Arabidopsis Gene Encoding a $\mathrm{Ca}^{2+}$-Binding Protein is Induced by Abscisic Acid during Dehydration," Plant \& Cell Physiology, vol. 41, no. 7, pp. 898-903, 2000.

[66] L. Sun, F. Song, J. Guo et al., "Nano-ZnO-induced drought tolerance is associated with melatonin synthesis and metabolism in maize," International Journal of Molecular Sciences, vol. 21, no. 3 , p. 782,2020 . 